\title{
Monitoring attention deployment by random number generation: An index to measure subjective randomness
}

\author{
FREDERICK J. EVANS \\ The Institute of Pennsylvania Hospital and \\ University of Pennsylvania, Philadelphia, Pennsylvania 19139
}

\begin{abstract}
The generation of random number sequences as a measure of attention deployment has been limited due to the lack of a satisfactory index of pseudorandomness for relatively short response sequences (Wagenaar, 1972). A new index of subjective randomization (RNG) is presented that provides a sensitive measure of departures from randomness (reflecting the disproportion with which any number follows any other number) in a series as short as 100 responses, typically verbalized at a rate of $1 / \mathrm{sec}$. The RNG index of sequential response bias is a minor modification of Tulving's (1962) subjective organization index that measures clustering in the repeated free recall of randomly presented word lists. Calculation of the practice-free RNG index and comparisons with another common measure are illustrated with individual data and with data obtained from random number tables. The use of RNG as a probe or simultaneous task to measure the deployment of attention or attentive effort is discussed.
\end{abstract}

There are more than 40 studies in the experimental literature in which the generation of random response patterns is the dependent variable. Although contradictory findings highlight this literature (Wagenaar, 1972), it is generally agreed that individuals are not good random generators. Hypotheses advanced to account for individual differences in the ability to randomize have stressed faulty concepts of randomness (Skinner, 1942), the facilitating (Tune, 1964) or inhibiting (Baddeley, 1966) effects of short-term memory, limited channel capacity for information processing (Baddeley, 1966), and the ability to either focus attention on (Weiss, 1964) or distract attention from (Wolitzky \& Spence, 1968) ongoing tasks. As Wagenaar (1972) stressed, a difficulty confronting previous investigators has been the multiplicity of often inadequate criteria for measuring the degree of subjective randomization in a reasonably short response series.

This paper reports the development of a new index of randomization derived from the subject's responses to being asked to generate random numbers using the digits from 1 to 10 inclusive. The method described

An earlier version was presented at the 18 th annual meeting of the Psychonomic Society, Washington, D.C., November 10, 1977. Supported in part by Grant MH19156-08 from the National Institute of Mental Health, U.S. Public Health Service, in part by the U.S. Army Medical Research and Development Command, Contract DADA17-71-C-1120, and in part by the Institute for Experimental Psychiatry. I wish to acknowledge the valuable technical assistance of Jeremy De Long, Charles Graham, Robert Hufgard, and David Paskewitz, and also thank David Dinges, R. Lynn Horne, John F. Kihlstrom, Pamela A. Markowsky, Emily Carota Orne, Martin T. Orne, Helen M. Pettinati, William M. Waid, and Stuart K. Wilson for their helpful comments. Request for reprints should be sent to Frederick J. Evans, 111 North 49th Street, Philadelphia, Pennsylvania 19139. appears to have several advantages: There are no apparent repeated performance practice effects; neither the subject nor the experimenter can easily evaluate how well the typical subject is performing; a stable index can be derived from a series as short as 100 numbers. The guiding hypothesis is that random number generation may provide a sensitive index of change in the deployment of attention over short intervals of time or the amount of attentive effort that is expended during a simultaneous task.

\section{DEVELOPMENT OF AN INDEX OF RANDOM NUMBER GENERATION}

\section{Rationale}

The index of randomness selected is a measure of sequential response bias. Intuitively, producing random numbers has some of the characteristics of a verballearning free-recall task, where the subject is required to repeatedly recall a list of words presented several times in a random order. Characieristically, subjects tend to impose an organizational structure on the word list to aid retrieval and recall, so that some words repeatedly cluster together.

Several procedures have been used to measure clustering or response sequences in free recall: The one most suited for adaptation to randomizing numbers is Tulving's (1962) Subjective Organization index. Tulving's procedure was directly adapted to measure sequential response bias in the production of what is analogous to the repeated free recall of a "list" of 10 numbers. The index presented below, and in Table 1, involves only a minor modification of Tulving's index: For its present application it has been relabeled the RNG index. 


\section{Procedure}

After starting a metronome, standardly set at $1 \mathrm{beat} / \mathrm{sec}$, the subject is instructed: "When told to begin, commence saying numbers aloud at the same rate as the metronome is beating $(1 / \mathrm{sec})$. Just repeat numbers in random order, using numbers from 1 to 10 inclusive. Certainly you are familiar with the concept of randomness. For example, if you were to throw a die many times, each of the six numbers would occur in random sequence. Although it might not happen in a few throws, over a large number of throws, each number would occur about as often as every other number, and in no particular order. Your task is to imagine tossing a 10 -sided die ... Say the numbers in random fashion... in time with the metronome... If you should find yourself ahead or behind the metronome, just try to get in pace with it again. Remember to use the numbers from 1 to 10 inclusive."

\section{Calculation of Random Number Index (RNG): \\ Ilustrative Example}

In response to these instructions, a female volunteer subject verbalized the following 100 responses $(1 / \mathrm{sec})$ in a baseline trial:

$\begin{array}{rrrrrrrrrr}7 ; & 1 ; & 5 ; & 8 ; & 3 ; & 4 ; & 10 ; & 9 ; & 6 ; & 2 ; \\ 1 ; & 4 ; & 7 ; & 9 ; & 5 ; & 2 ; & 3 ; & 10 ; & 8 ; & 6 ; \\ 7 ; & 2 ; & 1 ; & 5 ; & 4 ; & 9 ; & 6 ; & 1 ; & 10 ; & 4 ; \\ 2 ; & 3 ; & 7 ; & 8 ; & 9 ; & 4 ; & 5 ; & 6 ; & 2 ; & 10 ; \\ 8 ; & 9 ; & 4 ; & 3 ; & 7 ; & 5 ; & 6 ; & 1 ; & 9 ; & 8 ; \\ 3 ; & 4 ; & 6 ; & 10 ; & 9 ; & 8 ; & 7 ; & 2 ; & 3 ; & 1 ; \\ 9 ; & 4 ; & 5 ; & 7 ; & 10 ; & 6 ; & 9 ; & 2 ; & 8 ; & 7 ; \\ 3 ; & 5 ; & 1 ; & 6 ; & 4 ; & 3 ; & 9 ; & 5 ; & 6 ; & 1 ; \\ 8 ; & 10 ; & 7 ; & 5 ; & 9 ; & 2 ; & 4 ; & 3 ; & 8 ; & 1 ; \\ 9 ; & 7 ; & 6 ; & 3 ; & 2 ; & 7 ; & 10 ; & 9 ; & 5 ; & 1 .\end{array}$

These responses are tabulated in a 10 by 10 matrix, as shown in Table 1 . The matrix is arranged to reflect the frequency with which any number follows any other number in the 100 consecutive responses. This subject's first response, " 7, ," is followed by the response " 1 ," so a tally is entered at the intersection of the row (i) corresponding to " 7 " and the column (j) corresponding to " 1 ." The second response, " 1 ," is followed by the third response, "5," and a corresponding tally is made at Cell $i, j$ (i.e., the intersection of row 1 and column 5). In general, the nth response is cross-tabulated with the $(n+1)$ th response for each of the 100 responses in turn. (The 100th response, in this case " 1 ," is cross-tabulated against the initial response, " 7 ," for convenience, although it can be tabulated against 101 st response if it is available, with little or no effect on the magnitude of the index.)

In the series of 100 numbers, several sequences are repeated more than once. For example, the response "9" occurs a total of 10 times. When "9" was used as the subject's 35 th, 42 nd, and 61 st responses, it was followed by the response " 4 ." When "9" was used as the 14th, 77 th, and 98th responses, it was followed by the response " 5 ." Note that the response " 9 " is never followed by the response " $1 . "$ Therefore, the corresponding frequency in Cell ${ }_{9,4}$ is 3 , Cell 9,5 is 3 , and Cell 9,1 is 0 . However, Cell 1,9 frequency is also 3 . Thus, whenever this subject responded " 9 ," it was never followed by the response " 1 ," but when the 48th, 60th, and 90th responses were "1," each was followed by "9."

In a random sequence of 100 responses, each of the 10 numbers would theoretically follow each other number only once. It is this frequent usage of repeated pairs of sequences (such as " 9,4 "; " 9,5 "; " 1,9 " in the example) which builds up the value of the RNG index, calculated using the formula presented below and in Table 1 .

Following the tabulation of all such pairwise sequences marginal frequencies are determined by summation. The marginal frequency, $f_{1}$, of all responses of " 1 " is 10 , for all responses of " 9 " is 13. Theoretically, the expected marginal
Table 1

Illustrative Example of Randomization Matrix Used in the Calculation of the Random Number Generation Index (RNG) for a Volunteer Subject: Baseline Performance*

\begin{tabular}{|c|c|c|c|c|c|c|c|c|c|c|c|}
\hline \multirow[b]{2}{*}{$\mathbf{R}_{\mathbf{i}}$} & \multicolumn{10}{|c|}{$\mathbf{R}_{\mathbf{j}}$} & \multirow[b]{2}{*}{$\mathbf{f}_{\mathbf{i}}$} \\
\hline & 1 & 2 & 3 & 4 & 5 & 6 & 7 & 8 & 9 & 10 & \\
\hline 1 & & & & 1 & 2 & 1 & 1 & 1 & 3 & 1 & 10 \\
\hline 2 & 2 & & 3 & 1 & & & 1 & 1 & & 1 & 9 \\
\hline 3 & 1 & 1 & & 2 & 1 & & 2 & 1 & 1 & 1 & 10 \\
\hline 4 & & 1 & 3 & & 2 & 1 & 1 & & 1 & 1 & 10 \\
\hline 5 & 2 & 1 & & 1 & & 3 & 1 & 1 & 1 & & 10 \\
\hline 6 & 3 & 2 & 1 & 1 & & & 1 & & 1 & 1 & 10 \\
\hline 7 & 1 & 2 & 1 & & 2 & 1 & & 1 & 1 & 2 & 11 \\
\hline 8 & 1 & & 2 & & & 1 & 2 & & 2 & 1 & 9 \\
\hline 9 & & 2 & & 3 & 3 & 2 & 1 & 2 & & & 13 \\
\hline 10 & & & & 1 & & 1 & 1 & 2 & 3 & & 8 \\
\hline$f_{j}$ & 10 & 9 & 10 & 10 & 10 & 10 & 11 & 9 & 13 & 8 & 100 \\
\hline \multirow{2}{*}{\multicolumn{12}{|c|}{$\mathrm{RNG}=\frac{\Sigma\left(\mathrm{f}_{\mathbf{i j}}\right) \cdot \log \left(\mathrm{f}_{\mathbf{i j}}\right)}{\Sigma\left(\mathrm{f}_{\mathbf{i}}\right) \cdot \log \left(\mathrm{f}_{\mathbf{i}}\right)}$}} \\
\hline & & & & & & & & & & & \\
\hline \multicolumn{12}{|c|}{$=\Sigma(2 \log 2+3 \log 3+2 \log 2+\ldots 2 \log 2+3 \log 3)$} \\
\hline \multicolumn{12}{|c|}{$=\overline{\Sigma(10 \log 10+9 \log 9+10 \log 10+\ldots 13 \log 13+8 \log 8)}$} \\
\hline \multicolumn{12}{|c|}{21.6869} \\
\hline \multicolumn{12}{|c|}{$\overline{100.3376}$} \\
\hline & & & & & & & & & & & \\
\hline
\end{tabular}

Note-As $1 \log 1=0$, and thus where frequency of Cell $i, j$ is 0 or 1 , these do not directly enter calculations.

*Subject's 100 responses are tabulated in text.

frequency, $f_{1}$, for each of the 10 possible response categories or numbers should be 10 .

\section{Formula for RNG}

The formula for the index of randomization, after Tulving (1962), is:

$$
R N G=\left[\Sigma\left(f_{i j}\right) \cdot \log \left(f_{i j}\right)\right] /\left[\Sigma\left(f_{i}\right) \cdot \log \left(f_{i}\right)\right] .
$$

The numerator is a function of the sum of the log of all Cell $i, j$ frequencies; the denominator is a correction factor necessary when the obtained distribution of marginal cell frequencies deviates from (random) 10. The randomization index reflects the disproportion of sequence pairs within the cells adjusted by the disproportion of the marginal cell frequencies. It has a range of values from 0.0 to 1.0: A higher index reflects more extreme departure from the theoretical expected values, that is, it indicates poorer randomization. The RNG value for the data in Table 1 is .216 .

\section{THE RNG INDEX AS A MEASURE OF NONRANDOMNESS}

While all the expected frequencies in each matrix cell are equal to 1 (with 100 responses using 10 numbers), 
this is a theoretical distribution that would be approximated only by an infinite sequence of random responses: An index of 0.00 would be statistically highly improbable in a short series. Operationally, the RNG index measures nonrandomness or departures from randomness. The sampling fluctuations that could be expected in a 100-response series were tested by calculating the RNG value from 40 subsets drawn randomly from four sets of published random number tables. The mean randomness index was $.236(\mathrm{SD}=.025)$.

The main index previously preferred in studies of human randomization has been a derivative of the $H$ statistic drawn from information theory (Attneave, 1959). In terms of the matrix, in Table 1 the index $\mathrm{H} / \mathrm{H}_{\max }$ is calculated only from the marginal frequencies (usually of a longer string of responses). This value and the denominator of the RNG index are perfectly correlated. The RNG index and $\mathrm{H} / \mathrm{H}_{\max }$ correlated .34 in the 40 subsets of random number tables, indicating that the two indices are at least in part measuring different aspects of nonrandomness.

\section{Two Illustrative Examples}

Some of the properties of the RNG index can be illustrated with two sets of data from the same subject. The first, summarized above and in Table 1, was obtained from a standard baseline administration. This subject then generated random numbers while simultaneously performing a paper and pencil version of the difficult Stroop color-naming test (Messick, 1964), in which the subject is required to write down the color of the ink with which words naming a different color are printed (i.e., to write "red" when the word "green" is printed in red ink). During this simultaneous task, this subject's 100 random numbers were:

$\begin{array}{rrrrrrrrrr}1 ; & 3 ; & 5 ; & 7 ; & 6 ; & 8 ; & 5 ; & 3 ; & 2 ; & 1 ; \\ 7 ; & 6 ; & 5 ; & 4 ; & 2 ; & 1 ; & 3 ; & 7 ; & 10 ; & 9 ; \\ 8 ; & 6 ; & 5 ; & 4 ; & 3 ; & 2 ; & 1 ; & 7 ; & 6 ; & 5 ; \\ 9 ; & 10 ; & 6 ; & 5 ; & 10 ; & 10 ; & 10 ; & 3 ; & 2 ; & 1 ; \\ 10 ; & 9 ; & 8 ; & 7 ; & 6 ; & 4 ; & 3 ; & 7 ; & 5 ; & 10 ; \\ 9 ; & 8 ; & 6 ; & 7 ; & 9 ; & 8 ; & 3 ; & 2 ; & 1 ; & 3 ; \\ 2 ; & 6 ; & 5 ; & 9 ; & 8 ; & 2 ; & 1 ; & 4 ; & 10 ; & 4 ; \\ 5 ; & 7 ; & 10 ; & 9 ; & 8 ; & 7 ; & 10 ; & 1 ; & 2 ; & 3 ; \\ 5 ; & 9 ; & 3 ; & 2 ; & 1 ; & 6 ; & 7 ; & 8 ; & 9 ; & 3 ; \\ 9 ; & 2 ; & 1 ; & 3 ; & 7 ; & 5 ; & 4 ; & 3 ; & 2 ; & 1\end{array}$

The RNG index is .427 (see Table 2). Several changes in the Table 2 matrix are apparent. The number of cells with a zero frequency has almost doubled, and some cells have unusually high frequencies compared to Table 1 . While most of the diagonal cells have zero entries (subjects typically avoid repeating the same number, even though this kind of response sequence should by chance be as frequent as any other), the off-diagonal cells in Table 2 have very high frequencies. This represents repeated reverse counting (e.g., 7,6; 7,6,5;8,7,6; etc.): Counting in short strings (forward, backward, odd numbers, even numbers, etc.) becomes a typical strategy when a substantial cognitive stress is imposed during randomization.

The change in RNG of .216 to .427 from baseline to simultaneous Stroop test conditions represents a deterioration in randomization of about 4.5 standard deviation (z-score) units, based on parametric data from similar subjects. In contrast, the marginal frequencies, from which the previously favored $\mathrm{H}$ indices are calculated, are not noticeably different, and do not depart from the expected frequencies of $10\left(\chi^{2}=1.6\right.$,
Table 2

Illustrative Example of Deterioration in Randomization for the Same Subject Simultaneousiy Performing the Stroop Word-Color Interference Test*

\begin{tabular}{rrrrrrrrrrrr}
\hline & \multicolumn{10}{c}{$\mathbf{R}_{\mathbf{j}}$} \\
\cline { 2 - 8 } $\mathbf{R}_{\mathbf{i}}$ & 1 & 2 & 3 & 4 & 5 & 6 & 7 & 8 & 9 & 10 & $\mathbf{f}_{\mathbf{i}}$ \\
\hline 1 & 1 & 1 & 4 & 1 & & 1 & 2 & & & 1 & 11 \\
2 & 9 & & 1 & & & 1 & & & & & 11 \\
3 & & 7 & & & 2 & & 3 & & 1 & & 13 \\
4 & & 1 & 3 & & 1 & & & & & 1 & 6 \\
5 & & & 1 & 3 & & & 2 & & 3 & 2 & 11 \\
6 & & & & 1 & 5 & & 2 & 1 & & & 9 \\
7 & & & & & 2 & 4 & & 1 & 1 & 3 & 11 \\
8 & & 1 & 1 & & 1 & 2 & 2 & & 1 & & 8 \\
9 & & 1 & 2 & & & & & 6 & & 1 & 10 \\
10 & 1 & & 1 & 1 & & 1 & & & 4 & 2 & 10 \\
$f_{j}$ & 11 & 11 & 13 & 6 & 11 & 9 & 11 & 8 & 10 & 10 & 100 \\
\hline
\end{tabular}

Note- $R N G=.427$

* Subject's 100 responses are tabulated in text.

$p>.9 ; x^{2}=3.4, p>.9$, respectively, for $\mathrm{df}=9$ ). The corresponding $\mathrm{H} / \mathrm{H}_{\max }$ indices calculated from Tables 1 and 2 are .0034 and .0078 , respectively. The mean $\mathrm{H} / \mathrm{H}_{\max }$ values (which are highly correlated with $\chi^{2}$ ) derived from random number tables is $.0170(\mathrm{SD}=.0092, \mathrm{~N}=40)$.

The H-derived measure is not sensitive to the changes in randomization performance from Table 1 to Table 2 , and in fact suggests that both performances are slightly better than is obtained from random number tables. This impressionistic evidence, that RNG yields significant findings whereas the H-derived measures do not, has been repeatedly confirmed in our ongoing research.

\section{SOME PARAMETERS OF RNG PERFORMANCE}

In a number of samples varying in size from 10 to 84 , the mean RNG index, based on 100 numbers produced at $1 / \mathrm{sec}$, is about $.300(\mathrm{SD}=.045)$.

Retest reliability correlations vary between .40 and $.73(\mathrm{~N}=12, \mathrm{~N}=16)$ and tend to be higher if the first trial is excluded. The first trial is sometimes erratic; performance stabilizes as subjects presumably become adapted to the procedure as they try out a number of strategies, some of which they may reject as unsuccessful.

In one sample of 12 subjects, mean scores on three successive trials were $.292, .291$, and .294 , illustrating the lack of practice and sequence effects.

A number of factors have relatively small effects on RNG scores, in all cases being less than .5 of a standard deviation in size. Thus, slightly better randomization scores are found in males than in females, younger subjects, science rather than liberal arts majors, and subjects who are allowed to select their own performance speed (usually giving a response about every $1.7 \mathrm{sec}){ }^{1}$

\section{RNG AS AN INDEX OF ATTENTION DEPLOYMENT}

Two kinds of studies are currently being conducted to evaluate the validity of RNG as an index of attention 
deployment. As a baseline (single task) measure, it is being used to predict individual differences on other variables presumed to reflect attentional processes. For example, Graham and Evans (1977) reported that more hypnotizable subjects had significantly lower (more random) RNG indices than did less hypnotizable subjects. The randomization procedure should also prove effective as a probe or simultaneous task during other activities that require attentive effort. Graham and Evans (Note 1) asked subjects to perform the randomization task while simultaneously learning a two-hand motor coordination task. The initial deterioration of RNG from baseline, and then the gradual improvement as learning occurred, was a sensitive index of the acquisition process of the motor skill. Even when the motor task was being performed perfectly, RNG continued to recover, returning to baseline level only as overlearning led to automatic and effortless performance on the motor task. Other related studies in several areas, including hypnosis, pain, sleep, and a variety of cognitive and performance tasks in both normal and clinical populations, support the hypothesis that the RNG index is a sensitive measure of attentional processes.

\section{REFERENCE NOTE}

1. Graham, C., \& Evans, F. J. Subjective random number generation as an index of attention deployment. Paper presented at the annual meeting of the Eastern Psychological Association, Washington, D.C., May 1973.

\section{REFERENCES}

Atrneave, F. Applications of information theory to psychology. New York: Holt, Rinehart, \& Winston, 1959.
BADDELey, A. D. The capacity for generating information by randomization. Quarterly Journal of Experimental Psychology, 1966, 18, 119-129.

Graham, C., \& Evans, F. J. Hypnotizability and the deployment of waking attention. Journal of Abnormal Psychology, 1977, 86, 631-638.

Messick, S. Speed of color discrimination test. Princeton, N.J: Educational Testing Service, 1964.

SkINNER, B. F. The processes involved in the repeated guessing of alternatives. Journal of Experimental Psychology, 1942, 30, 495-503.

TulviNg, E. Subjective organization in free recall of "unrelated" words. Psychological Review, 1962, 69, 344-354.

TUNE, G. S. A brief survey of variables that influence randomgeneration. Perceptual and Motor Skills, 1964, 18, 705-710.

WAGENAAR, W. A. Generation of random sequences by human subjects: A critical survey of literature. Psychological Bulletin, 1972, 77, 65-72.

WeIsS, R. L. On producing random responses. Psychological Report, 1964, 14, 931-941.

Wolitzky, D. L., \& Spence, D. P. Individual consistencies in the random generation of choices. Perceptual and Motor Skills, 1968, 26, 1211-1214.

\section{NOTE}

1. Typically, subjects respond verbally with 1 response/sec. However, subjects can write their responses (preferably in a 10 by 10 matrix) without changing the basic randomization parameters. Different response rates (including unpaced) can be used, though randomization indices may vary when different rates are used or when the subject paces his own performance. A different number of response choices and a longer series can be used, though RNG parameters will then vary.

(Received for publication March 14, 1978.) 